\title{
¿SON ALGUNOS INTERFIJOS MORFEMAS APRECIATIVOS?
}

\author{
ARE SOME INTERFIXES APPRECIATIVE MORFEMES?
}

\author{
Antonio FÁbregas \\ UiT-Norges Arktiske Universitet \\ antonio.fabregas@uit.no
}

Recibido: 06/09/2016

Aceptado: 03/05/2017

\section{Resumen}

Este artículo se ofrecen varios argumentos para considerar que los interfijos y los morfemas apreciativos son en realidad la misma clase de objetos, como sugiere Lázaro Mora (1999), lo cual permite tratarlos unificadamente como marcadores de la no prototipicidad de lo expresado en la base. Entre las consecuencias de esta propuesta se encuentra que no pueden asociarse los diminutivos específicamente al cotejo del género de la base.

PALABRAS CLAVE: interfijación, morfología apreciativa, modificación, descomposición, flexión.

\begin{abstract}
In this article we offer a series of arguments that interfixes and appreciative morphology belong in fact to the same class of objects (as Lázaro Mora 1999 suggests); this allows for a unified treatment of both as markers of the non-prototipicity of that denoted by the base. Among the consequences of this proposal we find the fact that diminutives cannot be specifically associated to the checking of the gender of the base.
\end{abstract}

KEYWORDS: interfixation, appreciative morphology, modification, decomposition, inflection.

\section{EL PROBLEMA DE LA INTERFIJACIÓN}

El objetivo de este trabajo es el de argumentar que una clase de morfemas tratados como interfijos en la bibliografía (cf. Malkiel 1958; Alarcos 1983; Martínez Celdrán 1978; Montes Giraldo 1985; Miranda 1994; Bajo 1997; Almela 1999, 2015; Portolés 1999; Martín Camacho 2002; Varela 2005, entre otros) puede ser unificada con la clase de los afijos apreciativos.

Sin embargo, antes de entrar directamente en los argumentos a favor de proceder de esta manera se hacen necesarias algunas aclaraciones sobre el problema de la interfijación en español, ya que se trata de una cuestión polémica. Desde Lausberg (1949) -aunque sea

Para citar este artículo / To cite this article: Fábregas, Antonio (2017). ¿Son algunos interfijos morfemas apreciativos? ELUA, 31: 135-150. doi: 10.14198/ELUA2017.31.07

Enlace / Link: http://dx.doi.org/10.14198/ELUA2017.31.07 
más conocido el término a través de Malkiel (1958)- se ha observado que descriptivamente el español parece contar con una serie de morfemas segmentables que necesariamente aparecen en posición no terminal de la palabra, a los que se ha llamado 'interfijos'. Un caso clásico es el de (1).

\section{(1) vozarrón}

Por simple comparación entre palabras, las teorías que favorecen la segmentación de las palabras en morfemas entienden que es necesario aquí segmentar la raíz voz- y el sufijo -ón, aumentativo, pero queda dar cuenta del segmento -arr- que aparece entre ambos morfemas. En este punto, las teorías divergen acerca del tratamiento que debe darse a este segmento, y adoptan posturas distintas en virtud de dos parámetros:

a) La importancia relativa que se le dé a la evolución histórica de una forma frente a la interpretación que de ella haga sincrónicamente un hablante individual que puede no estar al tanto de las vicisitudes históricas que ha vivido una palabra.

b) La asociación entre forma y significado que se considere necesaria, aunque no suficiente, para darle carácter de morfema a un segmento dentro de una palabra.

En una primera aproximación al problema, las teorías que dan gran importancia a la evolución histórica de una forma notan que estos segmentos adicionales pueden explicarse como parte de la base o el sufijo en el interior de una palabra (Montes Giraldo 1985; Camacho 2002). Camacho (2002) discute el célebre caso de polvareda en este sentido, y observa que la afirmación de que -ar-debe tratarse aquí como morfema, junto a la raíz polv- y el colectivo -eda, olvida el hecho de que históricamente la palabra se forma sobre pulver-, lo cual en su criterio es suficiente para no tratarla como un afijo. Asimismo, si la teoría morfológica adoptada supone que las relaciones entre forma y signficado dentro de una palabra no son necesariamente locales -es decir, que cada morfema corresponda a un significado-, ya sea porque se adopta una visión morfológica paradigmática o porque se rechace la descomposición en morfemas como herramienta analítica, entonces tampoco surge la necesidad de segmentar morfemas con carácter de interfijos. El mismo Camacho (2002) propone que para explicar la presencia del segmento - $n$ - en formaciones como mironiano, daliniano o rousseauniano puede proponerse un proceso de estereotipia por el cual el segmento extra se toma analógicamente de otras formaciones más características de adjetivos derivados de nombres propios, como por ejemplo calderoniano, ciceroniano o moratiniano.

En cambio, las teorías que dan más importancia a la sincronía de una forma que a su evolución histórica, si además favorecen la descomposición en morfemas, proponen la existencia de interfijos para dar cuenta de segmentos como -arr- en vozarrón o -ar- en polvareda. A esta segunda clase de teorías pertenecen autores como Almela (1999, 2015), Miranda (1994) y Portolés (1999: 5044-5045). este último autor afirma que en la conciencia sincrónica del hablante la segmentación de estos segmentos es inevitable, pues de lo contrario nos obligaríamos a tratar el segmento -arrón como una variante alomórfica de -ón. Esto en sí mismo puede no ser problemático, pero tendría la consecuencia de que sería necesario diferenciar al menos cuarenta alomorfos de -ón, dada la serie de (2), lo cual sugiere que estaríamos perdiendo una generalización. 
(2) tem-erón, borb-ollón, mans-urrón, lim-atón, cosc-orrón, llam-arón, quer-endón, refunf-uñón...

La segmentación favorecida en estas teorías para evitar postular la existencia de tantas variantes alomórficas de un mismo sufijo tiene la consecuencia de que se hace necesario admitir que existen morfemas españoles que deben aparecer en posición intermedia de la palabra, los interfijos. Sin embargo, estas unidades son problemáticas para muchos autores por varios motivos.

a) En primer lugar, varios autores entienden que el interfijo es solo una unidad operativa del análisis gramatical, pero que no tiene realidad en la conciencia del hablante. Afirman estos autores - como Montes Giraldo (1985)- que el problema fundamental del interfijo es que no puede asociarse a un significado claro, lo cual entra en contradicción con los criterios estructuralistas de análisis morfológico. Esto lleva a algunos autores a proponer que el supuesto interfijo debe interpretarse como parte fosilizada de la palabra o como parte de un alomorfo fonológicamente condicionado de la base o del afijo (Martínez Celdrán 1978; Alarcos 1983; Bajo 1997). Nótese también que Pharies (2002) en su diccionario suele tomar la decisión de analizar casos de posible interfijación como variantes alomórficas de un afijo en el curso de la historia.

b) En segundo lugar, un problema al proponer que un afijo es interfijo es que existen numerosos casos en los que el segmento considerado interfijo posee otros usos en los que aparece como elemento terminal -solo seguido de las marcas de flexión-. En el caso de coscorrón, arriba notado, el segmento -orr- presumiblemente se relaciona con el mismo afijo que aparece en casc-orr(o). Tratarlo como un interfijo en el primer caso tiene la dificultad de que los afijos tienen posición fija, y en nuestro caso tendríamos un afijo que funciona tanto como interfijo como en calidad de sufijo, algo inusitado si consideramos las relaciones entre prefijos y sufijos, donde no se conocen casos en español donde un sufijo funcione en ocasiones como prefijo. Ante casos como estos, Camacho (2002) propone la posibilidad de que en algunas palabras se produzca la adición simultánea de dos sufijos independientes.

c) En tercer lugar, tenemos la dificultad de asignar un significado a muchos de los segmentos susceptibles de ser interfijos, lo cual es un lugar común en la bibliografía que trata estos elementos como incrementos fonológicos necesarios para satisfacer algunas condiciones de preservación de acentos, estructura silábica o choque de hiatos (Alarcos 1983; Varela 2005).

Concentrémonos ahora en este último problema: ¿cuál es la contribución gramatical y semántica de estos elementos? Malkiel (1958), de hecho, definió el interfijo como un "segmento siempre átono y falto de significado propio" (cf. Lázaro Carreter 1972), pero convertiría a los interfijos en una clase extraña de morfemas en español, caracterizados por ser los únicos que carecen de significado (Montes Giraldo 1985). Una alternativa dentro de la misma explicación general (Dressler 1986) ha sido que el interfijo tiene una función semántica indirecta, la de permitir reconocer la base al actuar como barrera fonológica que impida la fusión silábica entre la raíz y el sufijo. Almela (1999) propone, de hecho, que la cuestión no es que el interfijo carezca de significado, sino que no es posible limitar el do- 
minio del significado a los valores conceptuales de una palabra: para Almela el significado del interfijo es instrumental, dentro de la forma gramatical de la palabra, como engarce fonémico que habilita a una base para combinarse con otro elemento.

Con singular relevancia para este trabajo, Portolés (1988) trata también los interfijos como morfemas que se usan para habilitar una base para poder combinarse con un sufijo al que de otro modo no podría asociarse. Estudiando el caso de hum-ar-eda y polv-ar-eda, sugiere que en este caso concreto el interfijo -ar-se asocia semánticamente con una noción de 'abundancia' para poder formar un sustantivo colectivo a partir de una base que, por lo general, designa un nombre no contable. Dado que la idea de colectividad necesita la agregación de entidades para obtener un valor de abundancia, una base que denota un nombre no contable no puede asociarse directamente con un sufijo colectivo. El papel del interfijo es el de adaptar la base para permitir esta combinación y el de operar sobre el significado de la base para permitir la interpretación relevante, que también se da en hoj-ar-asca o vahar-ada. En muchos otros casos, el mismo interfijo se asocia a la noción de golpe o acción brusca, como es el caso de llam-ar-ada, uñ-ar-ada o lengu-ar-ada. Así, si bien el valor del interfijo es menos definido que el de un sufijo o un prefijo, también en este caso hay una contribución de significado que, en la línea de Aronoff (1976), se evalúa más claramente cuando se tiene en cuenta la palabra completa. El trabajo de Portolés sin duda convierte al interfijo - ar- en el mejor entendido de la gramática española, y partiremos de él para enunciar nuestra hipótesis.

En este trabajo tomaremos varias decisiones acerca de la clase de análisis que vamos a favorecer. En primer lugar, sin menoscabo de las explicaciones históricas, el presente trabajo se concentrará en la morfología sincrónica, y concretamente en tratar de entender de qué manera un hablante del español sin conocimientos etimológicos interpreta palabras como vozarrón o polvareda cuando se enfrenta a ellas. Es bien conocido que adoptar esta postura puede dar resultados opuestos a los que se obtienen cuando se emplea una metodología orientada a lo histórico. La NGLE (2009: §7.1f-j) habla de estos casos, entre los que cabe destacar, por su claridad, el del adjetivo asqueroso. Sabemos históricamente que este adjetivo existió antes que el sustantivo asco, que es una forma regresiva a partir del adjetivo (Miranda 1994). Sin embargo, desde una perspectiva sincrónica, un hablante que se enfrenta al adjetivo asqueroso tiende a relacionarlo con otros adjetivos en -oso que se forman a partir de sustantivos, como arenoso o pringoso. Sin presuponer que esta perspectiva sincrónica sea heurísticamente preferible en el análisis lingüístico, en este trabajo la adoptaremos.

En segundo lugar, como se puede comprobar, existen numerosos puntos de desacuerdo acerca de si existen los interfijos o, en caso de no existir, cómo deben tratarse. De hecho, la definición operacional que identifica los interfijos para los autores que creen en su existencia es particularmente vaga: son unidades segmentables, átonas, que aparecen entre la base y un sufijo o prefijo. Este criterio no permite deducir que todos los elementos que cumplan estas escasas propiedades formen una clase natural con propiedades positivas. Los afijos tienden a ser átonos, y en cuanto al criterio posicional, ya se ha visto que en muchos casos es discutible que deba aplicarse, puesto que hay unidades que parecen funcionar como sufijos y como interfijos. En este trabajo, precisamente por este motivo, trataremos de ser cautos y en lugar de proponer un análisis global de los interfijos que aspire a caracterizar sus propiedades en todas las situaciones, nos centraremos en una subclase de los segmentos que, en autores como Portolés (1999), se tratan como interfijos. Por tanto, lo que aquí se propone para la 
clase reducida que se discutirá no debe tomarse como una afirmación implícita de que los mismos criterios y propiedades se aplican a otros segmentos clasificables como interfijos, o incluso que en muchos de los casos de aparente interfijación el análisis sincrónico correcto no sea el de tratar dichos segmentos como interfijos. Así pues, con estas aclaraciones hechas explícitas, pasamos a la hipótesis que exploraremos en este trabajo.

\section{LA HIPÓTESIS: LOS INTERFIJOS VERBALES SON MORFEMAS APRECIA- TIVOS}

Como se ha visto, Portolés (1999) asocia el significado del interfijo -ar- a dos valores: abundancia y acción brusca. Tal vez el lector haya notado ya que estos dos significados -abundancia y acción brusca- están entre los que expresa típicamente el morfema aumentativo -ón.

(3) a. trip-ón, problem-ón

b. pescoz-ón, cap-ón

Es cierto que este morfema aumentativo comparte algunos significados con el homónimo -ón verbalizador que se documenta en palabras como empuj-ón, sin que esté clara la cuestión de si estamos ante un sufijo con varios valores o sufijos distintos que coinciden en algunos aspectos. Dejando esto de lado, superficialmente tenemos una coincidencia entre valores asociados a un interfijo y valores asociados a un morfema apreciativo. Podemos tomar esto como una coincidencia, o podemos tomarlo como evidencia preliminar de que aquí hay una generalización. En este trabajo, desde la perspectiva sincrónica notada, vamos a explorar la segunda vía, y vamos a proponer una hipótesis que resulta algo contraintuitiva pero que trataremos de argumentar en el resto del trabajo: los interfijos productivos del español son morfemas apreciativos, y forman una clase natural con los morfemas diminutivos, apreciativos y peyorativos. La hipótesis, por lo que sabemos, ha sido enunciada en forma inicial por Lázaro Mora (1999: 4646-4649). El objetivo de este trabajo es proporcionar una serie de argumentos que apoyan la propuesta unificadora de Lázaro Mora, cuyas consecuencias son potencialmente profundas para la comprensión de qué clase de elementos son los afijos que aparecen en el interior de algunos verbos derivados.

Para ello, por los motivos anotados en $\S 1$ sobre la falta de garantías de que todo segmento que se interpone entre la base y un afijo sea un morfema segmentable, nos concentraremos en solo un tipo de ellos: la clase de interfijos que aparecen combinados con formas verbales. La justificación de esta delimitación empírica es que para ellos, gracias al trabajo de Portolés (1988, 1999), su contribución semántica es algo más clara, y por lo tanto entendemos mejor sus propiedades, que pueden ser usadas para evaluar nuestra hipótesis. Nos referimos a los interfijos que se subrayan en (4) (cf. también Grandi 2008 para el italiano).

(4) a. dorm-it-ar

b. a-dorm-il-ar(se)

c. corr-et-ear

d. bes-uqu-ear

e. mord-isqu-ear

f. cant-urr-ear 
Junto a los argumentos que apoyan esta hipótesis, mostraremos que una visión unificada de los morfemas apreciativos y los interfijos permite avanzar en la comprensión de la restricción morfológica sobre su posición dentro de la palabra, específicamente por qué deben aparecer en posición interna y nunca como elementos iniciales o finales dentro de la palabra.

\section{PRIMER ARGUMENTO: IDENTIDAD MORFOLÓGICA}

Un primer argumento que apoya la propuesta es que una buena parte de los interfijos que se combinan con verbos son al menos homófonos con un sufijo apreciativo documentado.

\begin{tabular}{|c|c|c|}
\hline (5) a. (res)quebr-aj-ar & // & pequeñ-aj-o \\
\hline b. apret-uj-ar & // & bland-uj-o \\
\hline c. corr-et-ear & // & perr-et-e \\
\hline d. despat-arr-ar & // & chism-arr-o \\
\hline e. dorm-it-ar & // & gat-it-o \\
\hline f. chafarr-in-ar & // & mon-in-a \\
\hline g. apip-orr-arse & // & vent-orr-o \\
\hline h. bail-ot-ear & // & grand-ot-e \\
\hline i. bes-uqu-ear & // & tierr-uc-a \\
\hline j. escab-uch-ar & // & profesor-uch-o \\
\hline k. lamb-usqu-ear & // & verd-usc-o \\
\hline 1. a-lind-ong-ar(se) & // & mond-ong-o \\
\hline m. llor-iqu-ear & // & chiqu-ic-o \\
\hline
\end{tabular}

No todos los apreciativos se usan también como interfijos verbales (por ejemplo, -ón), ni todos los interfijos verbales se usan como apreciativos (por ejemplo, -asc- en churr-ascar), pero que la serie de equivalencias sea tan larga sugiere que no podemos confiar en que estamos ante una simple coincidencia fonológica. Una forma parsimoniosa de interpretar la larga serie de equivalencias de (5) es la de proponer que los afijos suenan igual en ambos casos sencillamente porque en ambos casos tenemos el mismo afijo. Es importante notar que la coincidencia fonológica no podría explicarse por el supuesto carácter no marcado o prototípico de los fonemas que componen estos afijos: encontramos combinaciones poco frecuentes, como -ng-, o formas no empleadas normalmente para resolver hiatos, como la consonante africada sorda o las vocales $/ \mathrm{o} / \mathrm{y} / \mathrm{u} /$.

Es relativamente fácil formalizar una situación en la que tenemos una sola clase de morfemas, que se usan tanto como interfijos verbales como en calidad de morfemas apreciativos. Muchos de ellos están subespecificados en cuanto a la clase de elementos a los que se unen (verbos o categorías nominales), pero algunos de ellos pueden contener especificaciones adicionales que requieran verbos o nombres y adjetivos. Esto daría cuenta de la situación ante la que estamos: muchos se emplean en ambos casos, pero algunos de ellos solo admiten verbos o clases nominales. La visión contraria, en la que las dos clases se encuentran completamente separadas, fuerza a tratar como una coincidencia las correspondencias de (5). 


\section{SEGUNDO ARGUMENTO: CONTRIBUCIÓN DE SIGNIFICADO}

Este argumento lo desarrolla Lázaro Mora (1999), apoyándose en observaciones de Rifón (1994) sobre la contribución semántica de los infijos. Rifón distingue tres valores fundamentales para los verbos con interfijación:

a) iterativo-habitual: repetición de una acción, o desarrollo interrumpido de una acción. Este uso es frecuente con -ot-, pero no exclusivo: palm-ot-ear, tir-ot-ear, parl-ot-ear, mord-isqu-ear.

b) intensivo-atenuado: o bien la acción se hace de forma intensa (bes-uqu-ear, tir-otear) o bien se hace de forma debilitada, solo aproximativamente a un modelo prototípico (dorm-it-ar, enamor-isc-ar, com-isc-ar, llor-iqu-ear)

c) peyorativo (llor-iqu-ear, mam-ull-ar).

Sin embargo, creemos que el valor semántico tiene que ser discutido desde otra perspectiva para delatar la correlación entre los valores de los interfijos y los de la morfología apreciativa. Un acercamiento que trate de identificar los mismos significados en uno y otro grupo se enfrenta a problemas serios, el menor de los cuales no es precisamente que un morfema apreciativo no tiene siempre un valor estable. Consideremos el caso de -ón, que se toma como prototipo del morfema que expresa exceso y aumento en español: junto a los valores aumentativos, hay al menos un caso en que la combinación de la base y el afijo da una interpretación de cantidad extrema, pero no por abundancia, sino por escasez:

(6) pel-ón

Tratar (6) como una forma lexicalizada tiene el inconveniente de que el adjetivo de (6) sigue hablando de tener una cantidad de pelo, y no otra noción. Para dar cuenta de esta restricción semántica es necesario admitir cierto grado de composicionalidad, y esto hace que el sufijo deba verse como un operador de cantidad extrema que no necesariamente especifica dicha cantidad como abundante.

De igual manera, con -ot-(e), se documentan casos en que la palabra denota una versión menor de la base (isl-ot-e). Por ello pensamos que el argumento semántico debe definirse de otra manera, y que así definido constituye realmente un factor adicional para considerar que las clases de los morfemas apreciativos y los interfijos deben unificarse.

Lo que observan tanto Rifón como Lázaro Mora es que en los derivados verbales por interfijación las tres dimensiones de significado mencionadas arriba tienden a solaparse. Por ejemplo, cabe interpretar lloriquear como una versión de llorar en que la acción se inicia, se abandona y se retoma varias veces (iteración), pero también como una forma de llorar que no llega a la intensidad prototípica de esta acción en ausencia del interfijo. Por último, se puede encontrar un valor peyorativo asociado a esta acción, o al agente de ella. Estos tres valores pueden aparecer simultáneamente en un texto, o uno de ellos puede destacarse frente al resto.

Esta misma propiedad, a saber, la de admitir diversas interpretaciones que se superponen, pueden convivir o pueden destacarse unas sobre otras, es típica de la morfología apreciativa. Es un lugar común (cf. NGLE 2009, por ejemplo) que es difícil discriminar entre 
valores peyorativos y aumentativos en muchos casos: decir de alguien que tiene un barrigón es tanto un juicio peyorativo como una descripción del tamaño de su vientre. En (7), como es bien sabido, cabe interpretar el diminutivo como una afirmación sobre el tamaño del bigote o como una evaluación emotiva de los sentimientos que despierta en el hablante (para bien o para mal) dicho objeto. En (8) cada una de estas interpretaciones, que se superponen sin cambio gramatical en (7), se destaca con la ayuda de otros modificadores.

(7) bigotito

(8) a. un bigotito casi imperceptible

b. ese dichoso bigotito

c. un adorable bigotito

Precisamente esta es la propiedad fundamental, creemos, de la morfología apreciativa: como han observado repetidamente autores como Dressler (1986), al agregar un morfema apreciativo a una base lo más que se puede decir es que estamos hablando de un valor no prototípico del significado de la base, tal que o bien la percepción subjetiva que tenemos de él es más relevante que el conjunto de propiedades objetivas que lo definen como predicado, o bien lo consideramos un exponente no típico de esa clase. La pragmática es la que determina en qué sentido esa base difiere de nuestra imagen prototípica de aquello a lo que debería corresponder.

A partir de aquí cabe hacer varias comparaciones. La acción de parlotear puede considerarse intensiva en la medida en que puede implicar un sentido de iteración y repetición abundante en un intervalo de tiempo (9a). No obstante, desde otra perspectiva, puede entenderse que el afijo contribuye un significado de atenuación: la acción de parlotear no solamente implica exceso en la cantidad de lo que se habla, sino que también parece asociarse a una falta en el contenido de lo que se dice (9b); no parece natural interpretar que una persona que da una conferencia densa sobre física cuántica, por mucho tiempo que ocupe su intervención, esté parloteando. Si se aplicara este verbo al conferenciante, entenderíamos que el hablante juzga que la intervención carecía de suficiente contenido.

(9) a. A Juan le gusta tanto parlotear que se le va la mañana entera sin trabajar.

b. Juan se pasó la reunión parloteando y nunca terminó de explicarnos qué quería que hiciéramos.

Es tentador asociar esta flexibilidad a casos en que el mismo diminutivo puede dar a entender grado elevado o grado reducido dependiendo del contexto pragmático, como en el caso del adverbio cerquita, que admite ambas interpretaciones.

(10) a. Está cerquita, no tardas nada en llegar (=muy cerca).

b. Está cerquita, pero no como para ir andando (=algo cerca, un poco cerca).

Considerado desde este nivel de abstracción, puede argumentarse que tanto los interfijos verbales como los morfemas apreciativos tienen la capacidad (muy probablemente entre otras) de tomar la noción que se expresa en la base con la que se combinan y presentarla en un grado mayor o menor del que se considera natural, de esta manera alejándolas de un modelo prototípico con el que se concibe esa base. Si concebimos la acción de comer como 
asociada a consumir cierta cantidad de alimentos, con cierta progresión temporal, frente a ella la acción de comiscar mostraría una situación que se aproxima a lo que consideramos comer, pero no de forma prototípica. De igual manera, lo que contamos como alto en cierto contexto no es lo que podríamos calificar como altito. En este sentido no existen otras clases sistemáticas de morfemas en español que se especialicen en este mismo papel. Hay ciertos prefijos que admiten lecturas de aproximación, como cuasi-, pero no es una propiedad sistemática de los prefijos expresar estos valores.

\section{TERCER ARGUMENTO: CAMBIO CATEGORIAL Y EXPRESIÓN DE LA MARCA DE PALABRA}

Es bien sabido que el análisis de la morfología apreciativa del español está complicado por el hecho de que los morfemas apreciativos, cuyo significado no es el esperable de la flexión, típicamente no cambian en español la categoría gramatical de la base, lo cual no es prototípico para la derivación tampoco. En español la morfología apreciativa puede tomar sustantivos y dar sustantivos (11), adjetivos para formar adjetivos (12) y adverbios para producir adverbios (13).
(11) reloj / relojito
(12) verde / verdecito
(13) lejos / lejitos

No obstante, hay casos esporádicos en los que el morfema apreciativo puede cambiar la categoría de la base. Portolés (1999: 5046) destaca -ón como un apreciativo que puede formar sustantivos a partir de verbos. Nótese que en las siguientes formaciones el valor de intensidad típico del aumentativo sigue presente, lo cual apoya un análisis en que no estamos ante un caso de homofonía.
(14) a. apag-ón
b. empuj-ón

Podemos añadir también el caso de -et-(e). Siendo un diminutivo, puede formar sustantivos a partir de verbos; nótese que los sustantivos así derivados se asocian al mundo infantil:
(15) a. jugu-et-e
b. chup-et-e

Lo que hace típicamente la morfología derivativa -sobre todo el diminutivo -it-(o)- es alterar el marcado morfológico de la base, forzando la introducción de una marca de palabra (Harris 1991) cuando la base no la tiene, o regularizando la marca de palabra a favor de la forma no marcada en español, que es $-a$ para las palabras femeninas y - o para las masculinas (Eguren 2002).

(16) a. *reloj-o

b. reloj-it-o 
(17) a. *man-a (man-o)

b. man-it-a

(18) a. *puent-o (puent-e)

b. puent-ecit-o

(19) a. *fuent-a (fuent-e)

b. fuent-ecit-a

(20) a. *Lourd-as (Lourd-es)

b. Lourd-it-as

Estas mismas propiedades morfológicas las encontramos sistemáticamente en el caso de los interfijos verbales. La primera propiedad destacable es que en la inmensa mayoría de los casos la interfijación no forma un verbo a partir de otra clase de palabras. En casi todos los casos concebibles estamos ante bases que ya pueden funcionar como verbos y que siguen siendo verbos una vez que se combinan con el interfijo. No obstante, como en la morfología apreciativa, hay también alguna excepción en que se puede producir el cambio categorial, y curiosamente una de ellas se refiere al morfema -et-, que en el caso de la morfología apreciativa ya hemos destacado como una excepción que sí permite cambio de categoría en la base.
(21) a. al-et-ear
b. tijer-et-ear
c. candil-et-ear

Si estamos ante el mismo afijo, este paralelismo deja de ser una coincidencia y pasa a ser una propiedad sistemática de -et-.

En segundo lugar, es bien sabido que la clase de conjugación por defecto, la no marcada, es la primera conjugación en español (Oltra-Massuet 1999). Por ejemplo, los neologismos verbales que adaptan préstamos del inglés sistemáticamente forman verbos de la primera conjugación (formatear, chequear, bloguear, twitear, googlear...). Pues bien: sistemáticamente, sea cual sea el interfijo empleado, los verbos con interfijo pertenecen a la primera conjugación incluso cuando la base pertenecía a cualquiera de las otras dos.
(22) a. *dorm-ar (dorm-ir)
b. dorm-it-ar
(23) a. *com-ar (com-er)
b. com-isc-ar
(24) a. *lam-ar (lam-er)
b. lam-isc-ar

Esto sugiere que en ambos casos estamos hablando de elementos que, en sentido amplio, no definen la clase categorial de la palabra, sino que la cotejan, es decir, entran en concordancia con ella, forzando la presencia de la forma no marcada para cada caso. Eguren (2002) relaciona la presencia de los diminutivos con el cotejo formal del género. Revisaremos la técnica de esta propuesta, cuya filosofía, en cambio, compartimos: la presencia de un diminutivo fuerza que la marca de palabra tenga el valor no marcado que se adapta a su valor de 
género, masculino o femenino. De la misma manera, en el verbo, la presencia del interfijo fuerza que su marca de palabra -en este caso, la vocal temática de conjugación- aparezca en su forma no marcada, que es la primera.

\section{CUARTO ARGUMENTO: LOS MORFEMAS APRECIATIVOS TAMBIÉN SUE- LEN SER INTERFIJOS}

Tal vez al examinar los ejemplos precedentes se haya notado ya que, de la misma manera que la marca de palabra debe quedar siempre a la derecha de los interfijos verbales, en el caso de los morfemas apreciativos siempre que la marca de palabra está presente se hace necesario que quede a la derecha del morfema apreciativo. Se repiten aquí varios de los ejemplos relevantes.
(25) a. pequeñ-ín-a
b. *pequeñ-a-ín
(26) a. vid-it-a
b. *vid-a-ít
(27) a. barrig-on-a
b. *barrig-a-ón

Nótese que no puede achacarse esto a un problema de fonotáctica que fuerce un ordenamiento determinado de los morfemas para evitar secuencias fonológicas imposibles: el español admite palabras con la forma final de (24b) (Mahón) o (22b) (Caín). Esta es una propiedad morfosintáctica, que aproxima en este sentido también los morfemas apreciativos a los interfijos.

\section{QUINTO ARGUMENTO: VARIABILIDAD}

Otro argumento es que tanto una clase como otra están sometidas a un considerable grado de variabilidad en la elección del morfema específico que se combina con cada base. Es bien sabido que un mismo hablante posee varias formas del diminutivo que, incluso sin tener en cuenta la variación geográfica, dan significados iguales con la misma base (28):
(28) a. perr-it-o
b. perr-et-e
c. perr-ill-o

De la misma manera, Portolés (1999: 5047) destaca que lo mismo puede encontrarse en el caso de los interfijos verbales.

(29) a. com-isqu-ear

b. com-istr-ear

(30) a. mam-ull-ar

b. mam-uj-ar 
La misma base admite varios interfijos en otros casos en que sí se dan diferencias de significado: a-dorm-il-ar vs. dorm-it-ar. El hecho sigue siendo que una raíz no fuerza necesariamente la presencia de un interfijo determinado, del mismo modo que no fuerza la presencia de un morfema apreciativo determinado. Esto contrasta claramente con el caso de otros morfemas del español: normalmente, una misma base verbal selecciona un sufijo y no otro para formar nominalizaciones:

(31) a. explic-a-ción (*explicamiento, *explicaje...)

b. mov-i-miento (*movición, *movaje...)

c. ancl-a-(a)je (*anclamiento, *anclación...)

Con los prefijos esto sucede también a menudo. Los temas griegos habitualmente niegan con la $a$-negativa, no con el prefijo in-. Ni en el caso de la morfología apreciativa ni en el de la interfijación se pueden hacer generalizaciones sistemáticas de esta clase entre la base y el morfema; en todo caso, se pueden hacer generalizaciones sobre el significado preferido para el conjunto de la palabra, como nota Portolés (1988), pero no se identifican relaciones locales de selección.
(32) a. a-morfo (*inmorfo)
b. á-crata (*incrata)
c. an-arquía (*inarquía)

\section{SEXTO ARGUMENTO: RECURSIVIDAD}

La morfología apreciativa es habitualmente citada porque permite cierto grado de recursividad en que morfemas que expresan la misma noción se pueden apilar unos sobre otros:

(33) chiqu-irr-it-ín

Portolés (1999: 5048) nota que entre los interfijos verbales se documentan también casos de series de interfijos donde se comprueba que varios segmentos susceptibles de ser analizados como tales se concatenan:

(34) pint-arr-aj-ear

(35) a-tont-ol-in-ar

(36) chap-al-et-ear

Esto sugiere que en ambos casos estamos ante elementos que, precisamente por no tener el papel prioritario de alterar la definición gramatical de la base, pueden unirse al resultado de haber sufijado con un elemento de la misma clase.

\section{CONCLUSIONES Y CONSECUENCIAS: HACIA UNA EXPRESIÓN UNIFICADA DE LA POSICIÓN LINEAL DE INTERFIJOS Y MORFEMAS APRECIATIVOS}

Los seis rasgos anteriormente mencionados para la clase de los interfijos verbales aportan cierta plausibilidad a la idea de que las dos familias de morfemas son realmente miem- 
bros de la misma clase, y esto permite avanzar hacia un análisis que explique las condiciones bajo las cuales un morfema tiene necesariamente que ocupar una posición intermedia entre la base y un sufijo o prefijo. Aquí solo esbozaremos un análisis que dé cuenta de las relaciones observadas, dado que el objetivo del trabajo es solo el de mostrar argumentos que apoyen la hipótesis de Lázaro Mora (1999).

Creemos que la propiedad fundamental que explica el orden de estos morfemas es que el español marca los temas morfológicos siempre con un elemento al que la bibliografía ha llamado 'marca de palabra' (Harris 1991, Piera 1995, Oltra-Massuet \& Arregi 2005). Esta marca aparece siempre en el margen derecho del tema morfológico y se relaciona con la categoría gramatical de la palabra.
(37) a. perr-o
b. bonit-o
c. cerc-a
d. cant-a

Oltra-Massuet (1999) ha argumentado que esta marca de palabra es siempre un incremento del morfema, nulo o con manifestación morfológica, que define la categoría gramatical de una raíz. Así, la representación de cant-a sería la de (38).

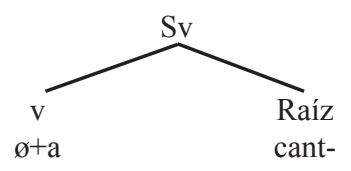

Portolés (1988) desarrolla la idea de que la función del interfijo es la de permitir que ciertos morfemas categorizadores se combinen con bases con las que, de otra manera, no pueden combinarse. La intención que tenemos en este análisis es la de mantener esta intuición. Concretamente, propondremos que un interfijo es un morfema carente de categoría gramatical que se genera como un modificador de la raíz.

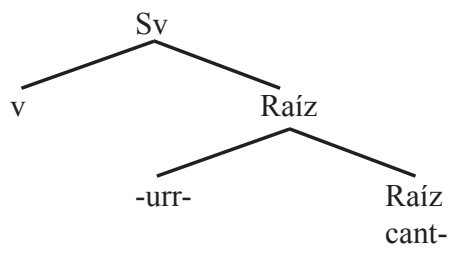

La estructura de (39) da, mediante las reglas de selección sintáctico-semánticas, una explicación al hecho de que no exista una selección directa entre la raíz y el interfijo. Como se puede ver en (39), el interfijo no es el complemento del verbo (v), sino un modificador del complemento de v. Es decir, si v selecciona un elemento, no será el interfijo, dado que este está contenido en su complemento pero no es el mismo complemento.

En segundo lugar, el carácter del interfijo como modificador en (39) explica que sea posible iterarlo. En cada iteración, un elemento vuelve a introducir sus rasgos en la estructura, lo cual tiene efectos para la clase de objetos que produce. El tiempo verbal, por ejemplo, es 
un núcleo que proyecta una serie de rasgos, y convierte el verbo base en un objeto de orden semántico superior que no denota solamente eventos, sino situaciones que contienen eventos. Pues bien: si un elemento es modificador, y por lo tanto no proyecta sus rasgos como núcleo, las iteraciones están permitidas porque ninguna de dichas iteraciones altera el tipo semántico o la etiqueta categorial del constituyente en que se dan. Por la misma razón, un sustantivo admite uno o más adjetivos, o un verbo admite uno o más complementos circunstanciales.

A partir de (39), el interfijo se desplaza a la proyección donde se define la categoría gramatical (Sv) para cotejar dicha categoría. Esto tiene dos consecuencias: la forma morfológica del marcador de categoría gramatical ahora es decidida por el interfijo, que está localmente más próximo que la raíz, y el cotejo fuerza la aparición de la forma por defecto del marcador de palabra.

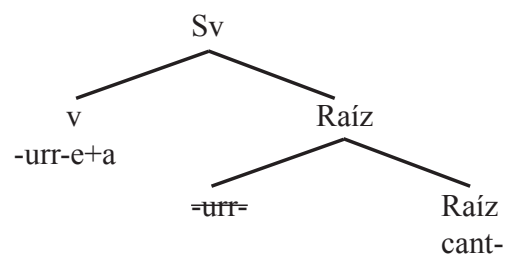

Eguren (2002) propone una operación de movimiento comparable, solo que en su caso el desplazamiento se produce para cotejar el género del sustantivo. Esto no podemos adoptarlo aquí, puesto que en el caso del interfijo verbal no habría género que cotejar, y es dudoso que, por ejemplo, los diminutivos con adverbios tuvieran valor de género. Adoptando su filosofía, pero no la implementación técnica, sería la necesidad de que el interfijo reciba categoría gramatical lo que lo llevaría a desplazarse hasta la proyección v. En el caso de -et-, que como vimos puede formar verbos a partir de sustantivos, proponemos que sucede exactamente lo mismo: el verbalizador es un morfo fonológicamente nulo, y el interfijo carece por sí mismo de categoría gramatical. Nótese que esto está forzado por los datos, dado que, de otro modo, esperaríamos que chupete fuera una forma verbal, y no un sustantivo.

Una vez que se ha producido esta operación, la raíz se incorpora al complejo formado ahora por el interfijo y el categorizador, dando lugar al orden linear correcto.

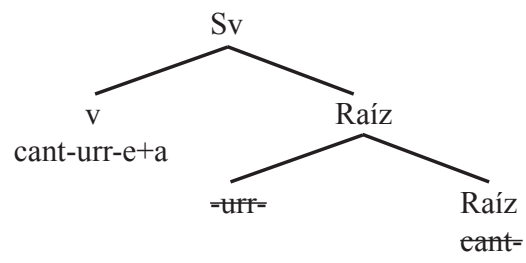

Estos son los pasos fundamentales que explican el orden linear en los interfijos verbales, y que proceden de forma parecida a lo que Eguren (2002) propone para los diminutivos. De esta manera, es posible explicar las restricciones de orden paralelas mediante la misma clase de operaciones, partiendo de los mismos primitivos. Naturalmente, esto solo es un esbozo de lo que debería ser un análisis completo de las formas, pero el objetivo de esta nota es solo el de mostrar que la hipótesis de Lázaro Mora (1999) tiene varios argumentos a su favor. 
En esta nota hemos decidido concentrarnos para formar el argumento sobre los interfijos que aparecen con los verbos. Como se ha explicado, hemos tomado esta decisión porque consideramos aquellos que se encuentran mejor descritos y entendidos en la actualidad. Queda por explorar de la misma manera los distintos tipos de interfijación nominal para comprobar si es posible tratarlos de una forma semejante a la que se ha mostrado aquí, o deben aceptarse otras explicaciones que tal vez confirmen las observaciones de autores como Camacho (2002).

También creemos necesario insistir en que la definición meramente posicional de interfijo esté definiendo como el mismo tipo de morfema a una clase que es potencialmente heterogénea: ciertos 'interfijos', discutidos por Lázaro Carreter (1972), sí tienen propiedades esperables de incrementos fonológicos destinados a disolver un hiato o preservar la coda silábica de otro morfema, como en café > cafe-t-ería, José > Jose-l-ito o ancho > en-s -anch$a(r)$ : no parecen aportar nociones sistemáticas de significado que modifiquen la semántica de la base, su aparición es mucho menos regular y los criterios que definen su distribución tienen propiedades fonológicas. Las reflexiones que se han apuntado aquí para la clase de los deverbales no se extienden, pues, a estos otros casos.

Sea cual sea el tratamiento analítico correcto para las clases de interfijos, esperamos al menos haber mostrado en estas páginas que no es descabellado incluirlos en la clase general de los apreciativos, por lo que no es necesario considerarlos morfemas excepcionales en español.

\section{Referencias bibliográficas}

Alarcos Llorach, Emilio (1983). "Consideraciones sobre la formación léxica". En Alarcos, E. et alii (eds.). Serta Philologica F. Lázaro Carreter I. Madrid: Gredos, pp. 11-15.

Almela Pérez, Ramón (1999). Procedimientos de formación de palabras en español. Barcelona: Ariel. Almela Pérez, Ramón (2015). Morfología léxica del español. Murcia: Diego Marín.

Aronoff, M. (1976). Word formation in Generative Grammar. Cambridge (Mass.): MIT Press.

Bajo, Elena (1997). La derivación nominal en español. Madrid: Arco/Libros.

Dressler, W. (1986). "Forma y función de los interfijos", Revista de la Sociedad Española de Lingüistica, 8, pp. 447-460.

Eguren, L. (2002). "Evaluative suffixation in Spanish and the syntax of derivational processes". En Herschensohn, J. et alii (eds.). Features and interfaces in Romance: Essays in the honor of Heles Contreras. Amsterdam: John Benjamins, pp. 71-84.

Grandi, N. (2008). I verbi deverbali suffissati in italiano. Cesena / Roma: Caissa Italia.

Harris, J. W. (1991). "The exponence of gender in Spanish”, Linguistic Inquiry, 22, pp. 27-62.

Lausberg, Heinrich (1949). Elemente der literarischen Rhetorik. Munich: Hueber.

Lázaro Carreter, F. (1972). "Sobre el problema de los interfijos: ¿consonantes antihiáticas en español?" En Lázaro Carreter, F., Estudios de lingüistica. Barcelona: Crítica, pp. 11-26.

Lázaro Mora, F. (1999). "La derivación apreciativa". En Bosque, I. y V. Demonte (dirs.). Gramática descriptiva de la lengua española. Madrid: Espasa, pp. 4645-4682.

Malkiel, Y. (1958). "Los interfijos hispánicos: problema de lingüística histórica y estructural”. En Catalán, D. (ed.). Miscelánea homenaje a André Martinet. La Laguna: Universidad de La Laguna, pp. 107-199.

Martín Camacho, J. C. (2001). "Sobre los supuestos diminutivos infijados del español”, Anuario de Estudios Filológicos, XXIV, pp. 329-341.

Martín Camacho, José Carlos (2002). El problema lingüistico de los interfijos españoles. Cáceres: Universidad de Extremadura. 
Martínez Celdrán, Eugenio (1978). "En torno a los conceptos de interfijo e infijo en español”, Revista Española de Lingüística, 8, pp. 447-460.

Miranda, J. Alberto (1994). La formación de palabras en español. Salamanca: Colegio de España.

Montes Giraldo, J. J. (1985). "Los interfijos hispánicos: reexamen con base en datos del ALEC", Anuario de filología hispánica, 1, pp. 181-189.

NGLE. RAE \& ASALE (2009). Nueva gramática de la lengua española. Madrid: Espasa.

Oltra-Massuet, I. (1999). "On the constituent structure of Catalan verbs". En Arregi, K. et alii (eds.). MIT Working Papers in Linguistics 33. Cambridge (Mass.): MIT Press, pp. 279-322.

Oltra-Massuet, I. \& K. Arregi (2005). "Stress-by-structure in Spanish", Linguistic Inquiry, 36, pp. 43-84.

Pharies, David (2002). Diccionario etimológico de los sufijos españoles. Madrid: Gredos.

Piera, C. (1995). "On compounding in English and Spanish". En Campos, H. y P. Kempchinsky (eds.). Evolution and revolution in Linguistics. Essays in Honor of Carlos Otero. Washington: Georgetown University Press, pp. 302-315.

Portolés, J. (1988). “Sobre los interfijos en español”. Lingüística Española Actual, 10, pp. 153-169.

Portolés, J. (1999). "La interfijación”. En Bosque, I. y V. Demonte (dirs.). Gramática descriptiva de la lengua española. Madrid: Espasa, pp. 5041-5073.

Rifón, A. (1994). "La habitualidad e iteratividad en la derivación verbal española", Verba, 21, pp. 183-206.

Varela, Soledad (2005). Morfología léxica: la formación de palabras. Madrid, Gredos. 